\title{
Le chercheur et la communication
}

\section{Hubert Curien}

\section{ADRESSE}

H. Curien : Ministre de la Recherche et de la Technologie. 1 , rue Descartes, 75231 Paris Cedex 05, France.

\section{TIRÉS A PART}

H. Curien.

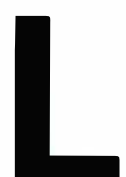

'activité scientifique relève à la fois de la création et de la fabrication. La découverte scientifique débouche sur la formulation d'un concept nouveau et cependant elle se nourrit de toutes les connaissances accumulées antérieurement et se rapportant de près ou de loin au sujet traité.

Le scientifique conçoit et réalise un programme expérimental, ou quelque calcul destiné à tester une hypothèse, cette démarche devant aboutir à une connaissance supplémentaire qui ne peut se réduire à une synthèse des savoirs existants ni au prolongement " naturel " de ce même savoir. La découverte scientifique s'élabore avec le souci permanent d'être accessible au reste de la communauté scientifique, à son tour prémisse possible pour de nouvelles découvertes. A cette fin, elle est proposée à l'ensemble des pairs sous la forme d'une publication qui doit permettre à n'importe quel chercheur, en n'importe quel lieu, de la reproduire avec succès.

On pourrait oser une métaphore où la presse scientifique serait ainsi comparée à un ensemble disparate de magasins, grands, petits, luxueux ou de troisième ordre où seraient proposés les résultats de la recherche. Dès lors, publier, chercher, et si possible trouver, sont parties intégrantes du métier de chercheur.

L'un des moteurs essentiels de la science est, à n'en pas douter, le progrès social : avancée des connaissances permettant l'augmentation du niveau moyen de conscience des citoyens, progrès des techniques et progrès économiques.
Outre la création et la production de nouveaux concepts, le chercheur a un devoir de communication avec la société qui l'entoure. Au premier chef, il y a la nécessaire confrontation avec les chercheurs des autres disciplines, car de même qu'il ne progresse que par l'acquisition et l'assimilation des connaissances de son champ disciplinaire, il a un besoin impératif des outils intellectuels ou techniques engendrés par d'autres disciplines, voire par d'autres domaines de la création intellectuelle. Il se doit, par ailleurs, de participer à l'information de la population dans son entier, par tous les moyens qui lui sont offerts : contacts directs, journalistes (presse spécialisée, médias généralistes), ou autres. Étape essentielle de cet accomplissement, le processus de valorisation industrielle est indiscutablement un moment clé, dont le produit le plus achevé est le brevet d'invention.

Enfin, on ne doit pas négliger une part importante de la matière imprimée dite "littérature grise " qui comprend, à l'exclusion des publications déjà évoquées, les thèses, DEA, rapports de mission et contrats de recherche...

\section{L'évaluation et la publication des résultats scientifiques : une situation chaotique}

L'évolution actuelle des organes de publications se caractérise de deux manières : l'inflation et le libéralisme sauvage.

Il y a une vingtaine d'années, dans 


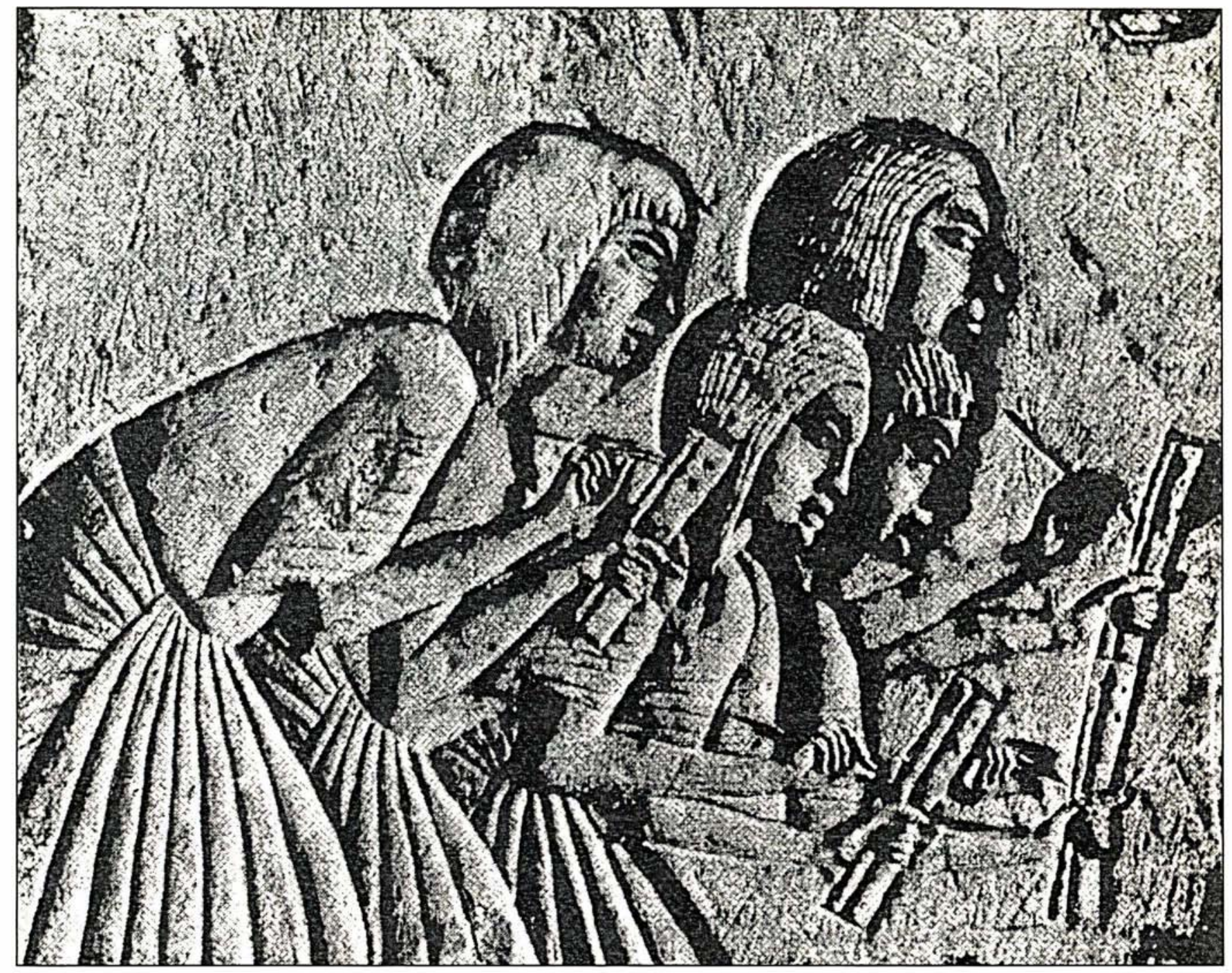

Scribe. (Gondal).

la plupart des disciplines scientifiques, existait un nombre restreint de revues, tout à fait vénérables, souvent organes officiels de sociétés savantes publiant une quantité limitée d'articles. L'évaluation était assurée par ce que l'on appelait "la revue par les pairs ", dont le principe consiste en une soumission de chacun des articles à des scientifiques reconnus dans leurs domaines. Ils se livraient à un examen attentif du manuscrit, vérifiaient sa validité, sa cohérence et son intérêt et donnaient leur aval, suggéraient des modifications ou, le cas échéant, refusaient sa publication.

A côté de ces revues spécialisées, inégales suivant la puissance et la noto$\mathrm{m} / \mathrm{s} n^{\circ} 3$ vol. 6 , mars 90 riété de la société éditrice, coexistait un petit nombre de revues généralistes qui publiaient des résultats considérés comme importants mais dépassant le cadre étroit d'une spécialité. Les archétypes de ce genre de revues sont Science et Nature.

Cette situation n'était naturellement pas idéale. En particulier, elle n'offrait pas de solution au problème du conformisme rencontré quelquefois dans les revues spécialisées. Il n'était pas toujours possible d'éviter un certain conservatisme d'équipes honorablement assises, manifestant quelque réticence à introduire dans leur cénacle de jeunes chercheurs aux démarches innovantes.

De ce point de vue, la situation s'est nettement améliorée avec l'essor, principalement en biologie, des nouvelles techniques, de l'accroissement des connaissances, de l'augmentation considérable du nombre des chercheurs et de l'émergence de nouveaux centres de recherche scientifique. La compétition entre les équipes a rendu nécessaire une publication plus rapide des résultats, même parcellaires.

Les étoiles montantes des nouveaux domaines scientifiques ont manifesté une certaine irritation devant les résistances qui leur étaient opposées par les honorables sociétés savantes dans la reconnaissance et la légitimation de leurs champs disciplinaires. Désireux d'ajouter à leur pouvoir 
scientifique tout neuf, un pouvoir éditorial, ils ont créé, et continuent aujourd'hui encore de créer, de nouvelles revues scientifiques.

Vigueur de la compétition, habitude prise de la publication précoce des résultats, augmentation des équipes de recherche, accroissement du nombre des revues, tout cela contribue à une inflation considérable de matériel publié. Cette augmentation est telle qu'elle dépasse même celle du nombre des "producteurs de la science ". La conséquence en est une perturbation profonde du sacro-saint système qui avait prévalu jusque-là, la "revue par les pairs".

Les habituels arbitres des articles scientifiques se sont vus submergés par une masse colossale de manuscrits à évaluer auxquels il n'a plus été possible dès lors d'accorder la même attention qu'auparavant. L'activité de recherche elle-même s'en est trouvée affectée par la multiplication déraisonnable d'informations scientifiques à consulter.

Grâce à des équipes dynamiques, de nouvelles publications sont devenues excellentes sans pour autant périmer les plus anciennes.

S'est accru aussi le nombre des revues généralistes publiant une part non négligeable d'articles scientifiques dans leurs colonnes.

Une des conséquences néfaste de cette situation fut cependant la prolifération de résultats insuffisamment étayés, parfois même rapidement infirmés, mais qui ont pu entraîner des chercheurs dans des impasses scientifiques.

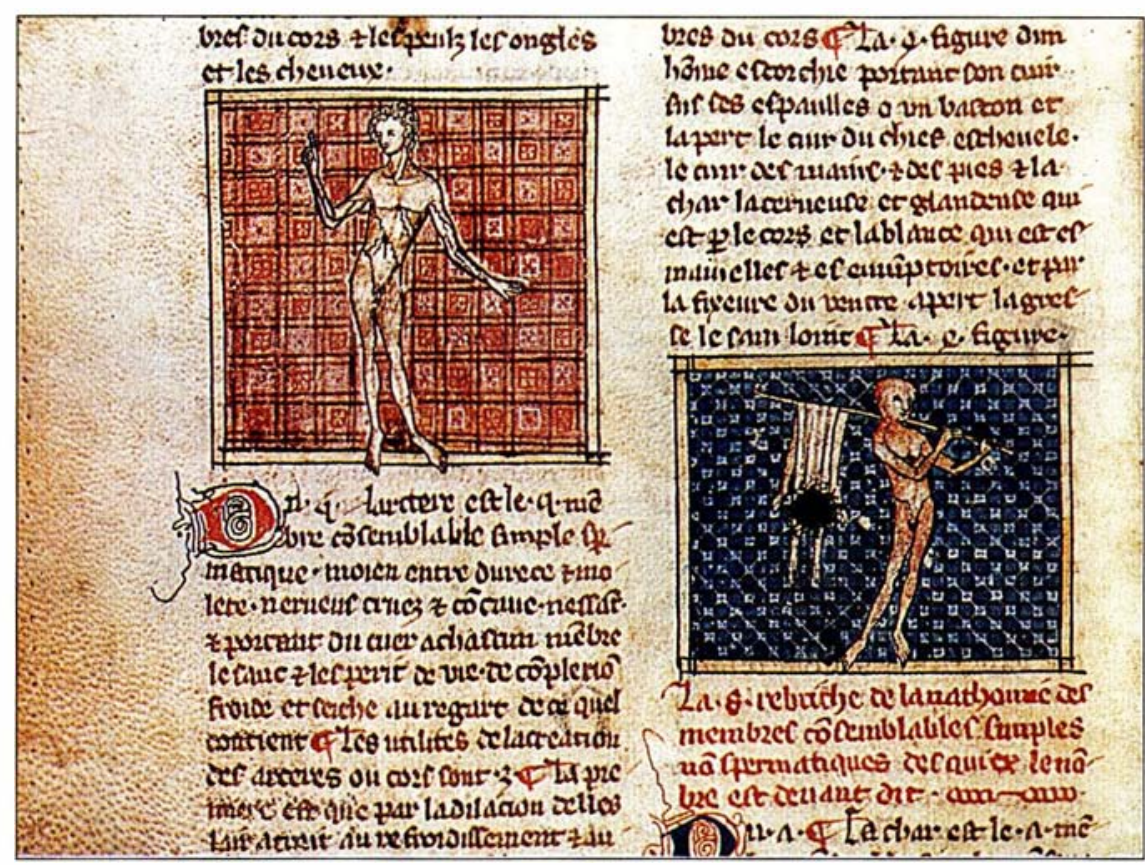

Manuscrit francais de 1314 de Henri de Mondeville, chirurgien de Philippe Le Bel et Louis Le Hutin. Henri de Mondeville est le premier chirurgien français ayant écrit un traité de chirurgie. IMs Fr. 2030, chirurgien de Mondeville, Bibliothèque Nationale de Paris).
D'autant plus qu'est même apparue une autre catégorie de publications dont l'existence semble ne se justifier que par l'activité de chercheurs dont les travaux n'ont pas abouti à la publication dans d'autres revues!

A maints égards, on peut craindre que la situation actuelle ne soit préjudiciable au bon développement des sciences

Il n'existe probablement pas de solution miracle pour stopper une telle évolution. La difficulté réside dans le fait que tout essai de réforme du système actuel de publication se doit d'obtenir un minimum de consensus international. Néanmoins, certaines pistes peuvent être ouvertes. Le développement des moyens informatiques, la capacité de stockage des données, laissent envisager la possibilité que certains résultats expérimentaux, éventuellement significatifs mais ne justifiant pas une publication complète, soient accessibles à la communauté scientifique par le biais d'un système élaboré d'interconnexions et d'interrogations par mots clés.

Un nombre plus restreint de grandes revues thématiques, parmi lesquelles des revues européennes, publierait les résultats de travaux parvenus à un degré de maturation suffisant. Des journaux pluridisciplinaires, tels que Nature ou Science, conserveraient pour prérogative la publication rapide de résultats apparemment essentiels dans divers domaines scientifiques. Parallèlement, il serait bon que les instances chargées du recrutement et de l'évaluation des chercheurs incitent ces derniers à modérer leurs ardeurs "publicitaires" au profit de travaux plus accomplis.

\section{La communication scientifique interdisciplinaire : la place des cultures nationales}

Les revues les plus prestigieuses émanent des groupes les plus influents. Pour l'immense majorité d'entre elles, elles sont d'origine anglosaxonne. Dans le même temps, la langue utilisée pour les communications scientifiques est celle que la majorité des scientifiques ont en commun, c'est-à-dire l'anglais. Or, la langue de publication des résultats 
primaires de recherche doit privilégier l'intelligibilité par le plus grand nombre. Dans ce sens, et en l'état actuel du monde, l'anglais demeure une langue favorisée. Le constater n'est pas s'en réjouir et n'éteint pas le désir de voir la science française occuper au niveau mondial toute la place que le génie créateur de ses scientifiques est en droit d'espérer.

Les mouvements de la création scientifique s'intègrent à une activité intellectuelle élargie où les disciplines se nourrissent les unes des autres et s'enrichissent même d'autres champs de la connaissance. Il est donc primordial d'explorer les modalités possibles de communication entre chercheurs d'une même discipline ou de disciplines différentes. Ce dialogue interdisciplinaire devrait contribuer à forger l'outil cognitif de la création scientifique et pouvoir bénéficier de la souplesse, de la subtilité que seule la langue maternelle est à même de fournir. La pensée scientifique est partie intégrante de la culture nationale. Un pays qui perdrait toute possibilité d'énoncer dans sa langue les progrès de l'esprit, dans un domaine d'activité aussi primordial que la science, s'engagerait dans la voie dangereuse de la paupérisation culturelle et du déclin.

Il faut qu'existe une catégorie de publications scientifiques qui permettent aux chercheurs des différentes disciplines de conforter, dans leur langue, leurs concepts et leurs analyses critiques des évolutions de leur domaine. Permettre aux communautés nationales de se réapproprier le langage des sciences constitue un irremplaçable outil de formation à la pensée et à la pratique scientifique. médecine/sciences constitue, dans le domaine de la biologie et de la médecine, un bon exemple de ce type en France.

\section{Les sciences et la société}

Le développement constant des connaissances scientifiques et leurs applications technologiques crée un hiatus entre la science et les citoyens. Il s'ensuit, dans nombre de sociétés occidentales, un mouvement bien perceptible aujourd'hui de contestation de la démarche scientifique, appréhendée comme une tentative illégi- bres naturels. Peu ou mal informés de la réalité scientifique, inquiets de ce qu'ils croient en comprendre et de ce qu'ils en imaginent, un certain nombre d'entre eux prêtent une oreille complaisante à un courant de pensée profondément anti-scientifique. Quant aux chercheurs, ils sont peu enclins à diffuser et à expliquer leurs résultats au grand public. Il faut dire, à leur décharge, qu'ils n'y sont pas incités par les méthodes d'évaluation de leurs travaux qui prennent trop peu en compte les efforts d'information et de vulgarisation.

L'attitude des médias, qui trop souvent n'accordent d'intérêt qu'aux aspects les plus artificiellement spectaculaires de la science, n'incite pas non plus les chercheurs à se rapprocher des journalistes. Il y a pourtant nécessité de faire participer les scientifiques à la formation et à l'information du grand public. En France existent d'excellentes revues, à destination d'un public cultivé, comme, par exemple, La Recherche. Certains médias d'information possèdent des journalistes scientifiques de talent. Reste à valoriser l'apport des chercheurs à cet effort de vulgarisation en direction de publics d'âges et de niveaux culturels différents.

\section{Publication et brevet}

Il est légitime qu'un gouvernement attende de l'activité de recherche des atouts supplémentaires, pour l'industrie en particulier et l'économie plus généralement. Cependant les règles encore en vigueur en Europe placent le chercheur devant un dilemme : la publication ou le brevet. Dans ces conditions, beaucoup de chercheurs à vocation académique négligeront la prise d'un brevet potentiellement important pour leur pays si cela doit retarder une publication, but de tous leurs efforts, alors qu'à l'inverse, beaucoup de chercheurs impliqués dans l'industrie s'abstiendront de publier des résultats couverts potentiellement par un brevet.

Dans une certaine mesure, le système américain évite cet écueil en prescrivant un délai de grâce d'une année entre la date de publication et la prise d'un brevet. En d'autres termes, une équipe peut publier ses travaux en conservant la possibilité de les couvrir par un brevet dans l'année qui suit. Le système européen aurait intérêt à s'inspirer de ce principe afin de diminuer les réticences des trop nombreux chercheurs publics qui se refusent à prendre des brevets et augmenterait notablement le nombre des chercheurs industriels qui participeraient par leur publication à l'évolution des connaissances scientifiques mises à la disposition de tous.

Le constat qui vient d'être fait sur l'état de la communication scientifique entre pairs, à l'intention des chercheurs de disciplines différentes ou de la société en général, révèle une crise de nature à freiner le développement des sciences elles-mêmes et à entraver la progression de la conscience collective que l'on est en droit d'attendre de la science. En conséquence, dans une période où tout s'est emballé, et pour éviter, comme le disait le Président de la République, que la " science n'aille plus vite que les hommes ", il faut imaginer de nouvelles formes de communication. Celles-ci doivent prendre en compte les nouvelles conditions de production des résultats scientifiques. Il faut que soit assurée une nécessaire information pluridisciplinaire des chercheurs qui ont déjà quelque mal à suivre l'information de leur propre secteur de recherche et n'ont pas toujours l'opportunité de s'informer convenablement de ce qui se passe dans les disciplines voisines.

L'informatique, les moyens modernes de stockage et de diffusion des données, constituent des outils précieux pour faire face à la crise actuelle de diffusion de l'information scientifique. On ne dira jamais assez combien il est indispensable que les chercheurs participent à la formation des publics et au processus de vulgarisation de l'information scientifique, afin que soit évité un déphasage, un malentendu, qui pourrait devenir inquiétant entre les scientifiques et le grand public. L'importance de ces divers chantiers explique l'intérêt et le soutien des pouvoirs publics pour toute tentative originale qui tenterait de répondre, à quelque niveau que ce soit, aux questions soulevées par les insuffisances de la communication scientifique dans le contexte actuel d'évolution des sciences et des techniques

$\mathrm{m} / \mathrm{s} n^{\circ} 3$ vol. 6 , mars 90 